\title{
Secondary Infection/Microbial Substitution in a Managed Case of Pyogenic Spondylitis
}

\author{
Kengo Fujii ${ }^{1}$, Toru Funayama ${ }^{2}$, Sayori Li $^{1}$, Masashi Yamazaki ${ }^{2}$ \\ 1. Orthopedic Surgery, Showa General Hospital, Kodaira, JPN 2. Orthopedic Surgery, Faculty of Medicine, University of \\ Tsukuba, Tsukuba, JPN
}

Corresponding author: Kengo Fujii, kengox15feb@gmail.com

\begin{abstract}
Pyogenic spondylitis is a challenging condition that requires early and accurate diagnosis for appropriate treatment. Most cases can be treated non-surgically or with minimally invasive surgical procedures; however, a combination of anterior debridement/bone grafting and posterior fixation is necessary for severe cases. We encountered a case of lumbar pyogenic spondylitis treated with anterior debridement and autogenous bone grafting after percutaneous endoscopic discectomy drainage (PEDD) with percutaneous pedicle screw (PPS) fixation. The continuous pus oozing from the PEDD drainage tube wound was characteristic in this case, and the pus was considered to be caused by secondary infection/microbial substitution. The discharge immediately stopped and healed after anterior debridement and autogenous bone grafting. Escherichia coli was first detected as the causative bacterium, and Corynebacterium amycolatum and Corynebacterium striatum were detected as the cause of secondary infection/microbial substitution. The possibility of secondary infection/microbial substitution should be considered when the clinical course worsens.
\end{abstract}

Categories: Infectious Disease, Orthopedics

Keywords: pyogenic spondylitis, percutaneous endoscopic discectomy, posterior percutaneous fixation, anterior debridement fusion, secondary infection

\section{Introduction}

Pyogenic spondylitis (PS) is a challenging condition; immediate and accurate diagnosis followed by appropriate treatment are essential. Treatment options for PS include conservative therapy, rest, and antibiotics for most cases; however, surgical intervention is required for intractable cases, such as progressive neurological deficits, spinal instability, destructive changes, and worsening pain despite adequate antimicrobial therapy [1,2]. Percutaneous drainage is a minimally invasive option [3]. Percutaneous endoscopic discectomy drainage (PEDD) is useful because it is minimally invasive and enables curettage of the infected area under visualization and simple puncture and drainage tube insertion [4-10]. Posterior percutaneous pedicle screw (PPS) fixation is a minimally invasive technique useful for infectious spinal conditions [11-13]. Anterior debridement and autogenous bone grafting are widely performed to achieve both sufficient debridements of the infectious site and stabilization [14-16]. For most cases, PS can

Review began 06/22/2021 Review ended 07/03/2021 Published 07/16/2021

\section{() Copyright 2021}

Fujii et al. This is an open access article distributed under the terms of the Creative Commons Attribution License CC-BY 4.0., which permits unrestricted use, distribution, and reproduction in any medium, provided the original author and source are credited. be treated with non-surgical procedures or minimally invasive surgical procedures; however, a combination of anterior debridement/bone grafting and posterior fixation is necessary for severe cases.

We report a case of lumbar PS treated with anterior debridement fusion after PEDD and PPS fixation because of the continuous oozing of pus from the postoperative PEDD incision resulting from secondary infection/microbial substitution.

\section{Case Presentation}

A 78-year-old man experienced fever and back pain five weeks before his first spinal surgery. He did not have any history of diabetes, use of steroids, or any other immunosuppressive conditions instead of gastric cancer. He visited a local clinic two days after onset and was diagnosed with choledocholithiasis cholangitis. He was transferred to our institute the next day for gastrointestinal endoscopic treatment; however, this treatment was unsuccessful because of his previous surgery for gastric cancer. He underwent emergency open cholecystectomy and lithotripsy for common bile duct stones on the same day. After abdominal surgery, fever and back pain continued with high C-reactive protein levels (range: 4.1-20.8 mg/dL), despite intravenous administration of antibiotics (Figure 1). First, tazobactam/piperacillin (TAZ/PIPC) was chosen as an empiric broad antibiotic. It was de-escalated to ceftriaxone (CTRX) after Escherichia coli (E. coli) was detected in the results of two consecutive blood culture tests (Figure 1). Multiple penicillin and cephem derivatives were sensitive to E. coli. Enhanced computed tomography (CT) images to determine the source of inflammation at 11 days after admission revealed highly enhanced signal changes in the L3/4 intervertebral disc and a right psoas muscle abscess connected to the L3/4 disc space (Figure 2). Lumbar magnetic resonance imaging (MRI) showed low-signal changes in the caudal L3 and cranial L4 vertebral bodies on T1weighted images, high-signal changes on T2 fat-suppressed images, and enhanced gadoliniumdiethylenetriamine pentaacetic acid (Gd-DTPA) on T1-weighted images (Figure 3). We chose conservative 


\section{Cureus}

therapy and continued intravenous CTRX administration (Figure 1). Four weeks after admission, his back pain did not improve and C-reactive protein levels began to increase. Lumbar MRI and CT scans showed progressive destructive changes in the L3 and L4 vertebral bodies (Figures 4, 5).

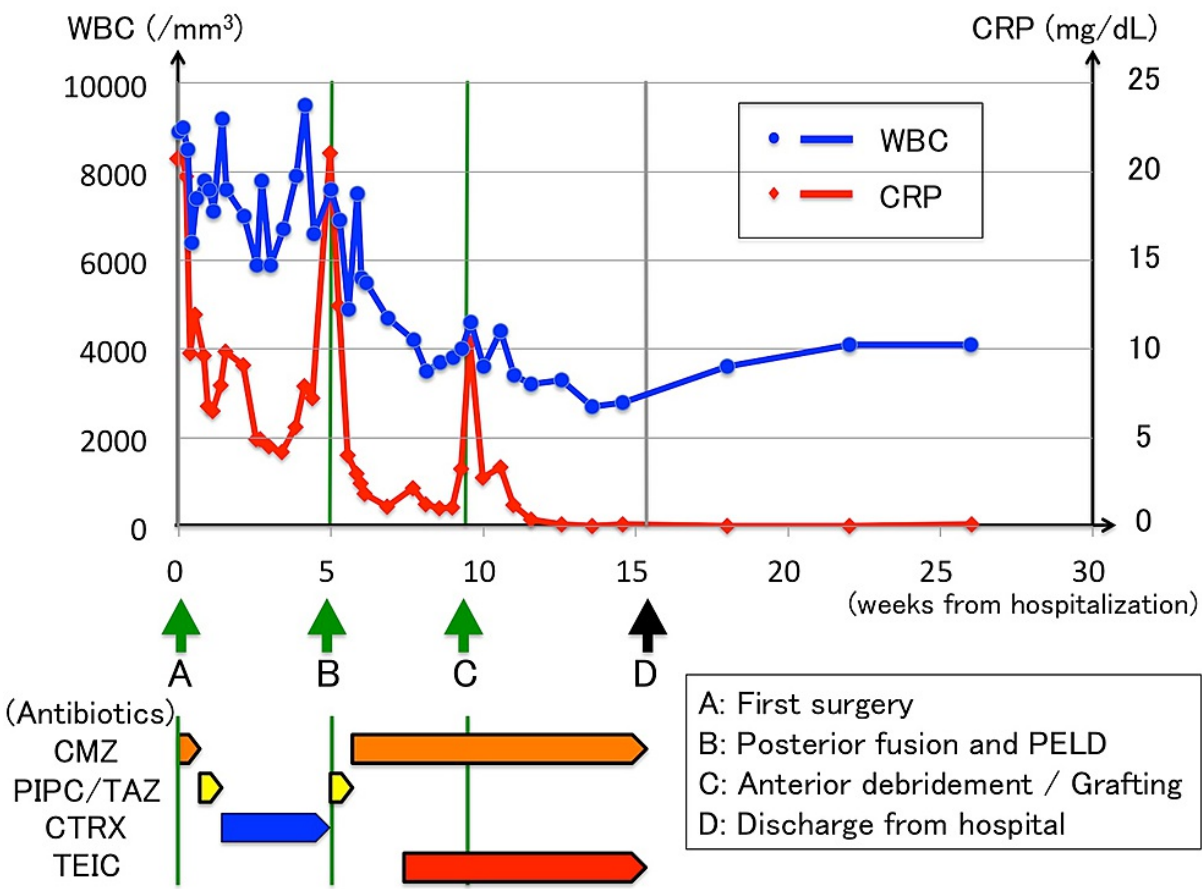

FIGURE 1: Clinical course and antibiotic administration schematic and graph of the blood test results.

The graph shows the changes in the laboratory data, including the white blood cells (WBC) and C-reactive proteins (CRP). Bars and arrows below the graph show the time points of antibiotic administration: (A) first surgery; (B) posterior fusion and percutaneous endoscopic lumbar discectomy/debridement (PELD); (C) anterior debridement/grafting; and (D) discharge from hospital.

CMZ, cefmetazole sodium; TAZ/PIPC, tazobactam/piperacillin; CTRX, ceftriaxone sodium; TEIC, teicoplanin

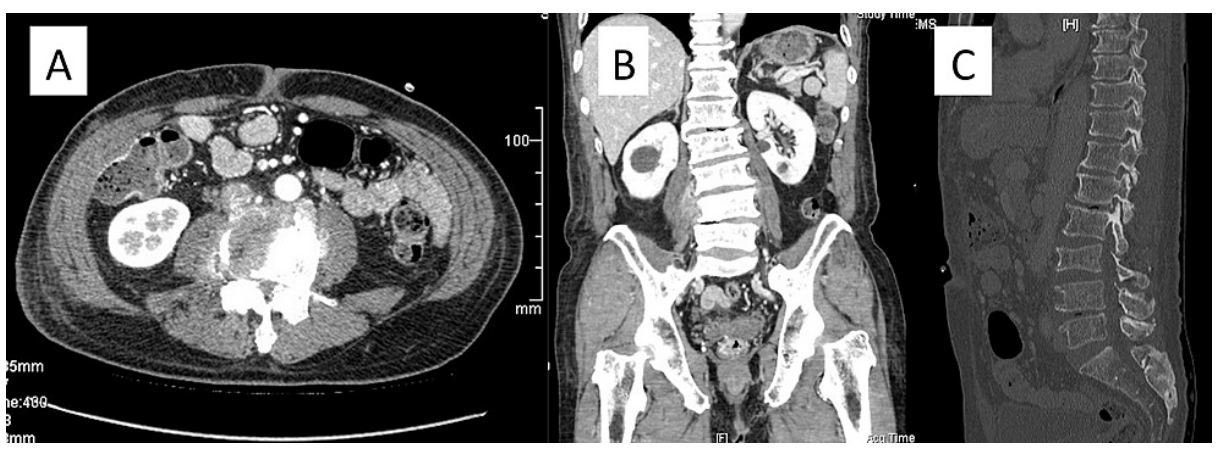

FIGURE 2: Enhanced computed tomography scan slices.

(A) Axial slice shows an enhanced lesion around the L4 vertebral body and right psoas muscle, suggesting the focus of infection. (B) Coronal slice shows an enhanced lesion in the right psoas muscle, suggesting psoas abscess. (C) Sagittal bone quality slice shows no destructive changes in the L3/4 endplates and vertebral bodies. 


\section{Cureus}

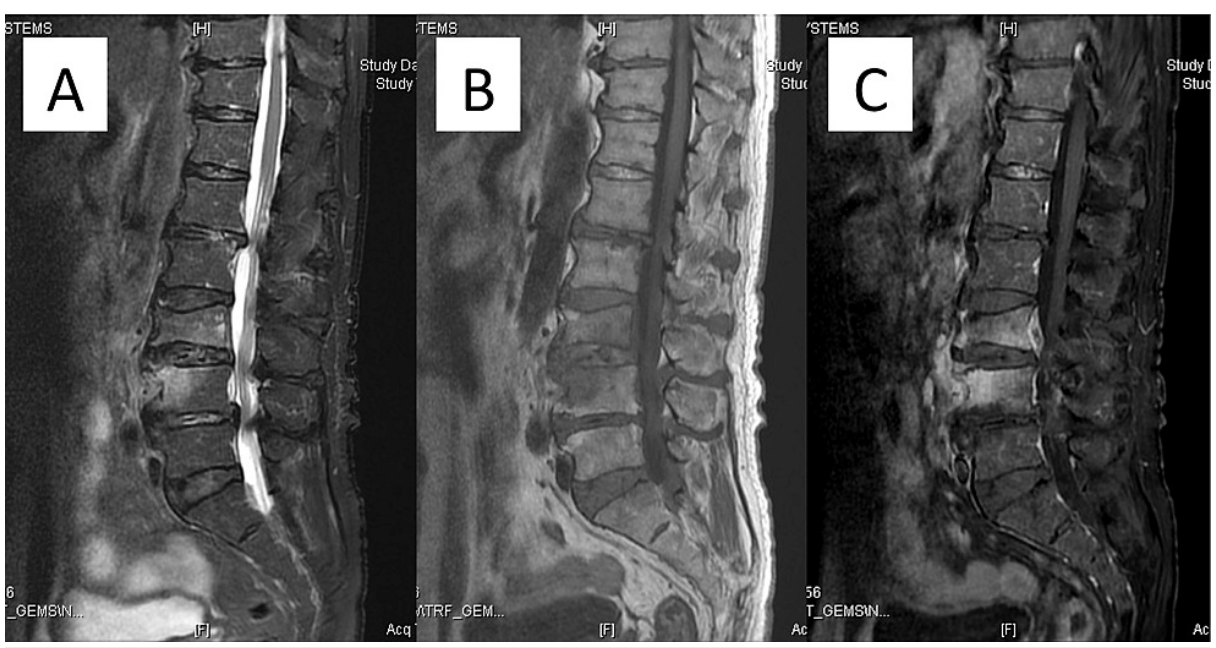

FIGURE 3: Gadolinium-diethylenetriamine pentaacetic acid (Gd-DTPA)enhanced lumbar images obtained with magnetic resonance imaging.

(A) Sagittal T2-weighted fat-suppressed image shows high-intensity changes in the L3 and L4 vertebral bodies. (B) Sagittal T1-weighted fat-suppressed image shows low-intensity changes in the L3 and L4 vertebral bodies. (C) Sagittal Gd-DTPA-enhanced T1-weighted image shows enhancement in the L3 and L4 vertebral bodies, anterior paravertebral soft tissue, and epidural abscess at the L3 vertebra level.

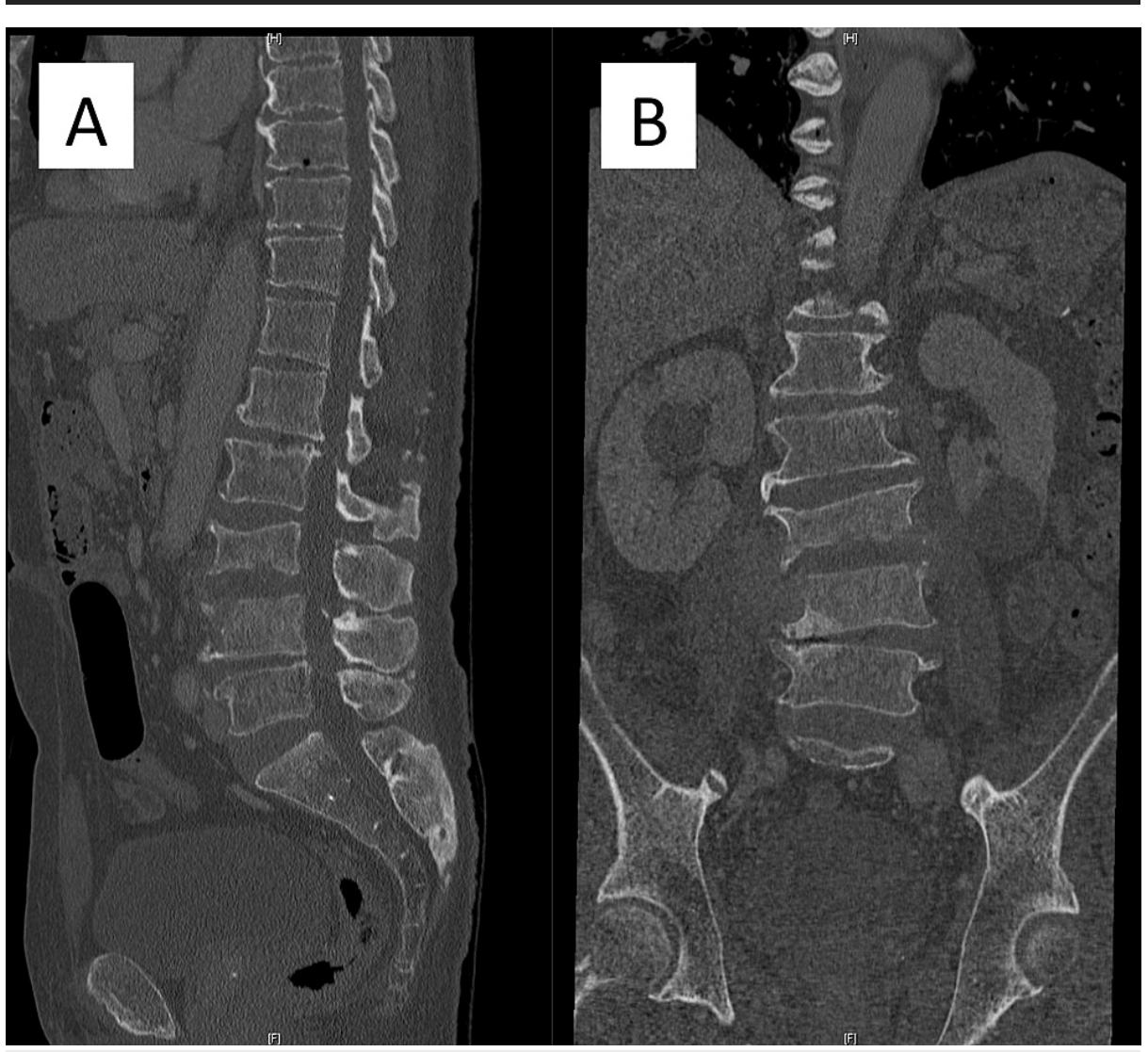

FIGURE 4: Secondary computed tomography scan slices after four weeks of antibiotics administration.

(A) Sagittal slice. (B) Coronal slice. Progressive destructive changes of the L3 and L4 endplates and vertebral body were observed. 


\section{Cureus}

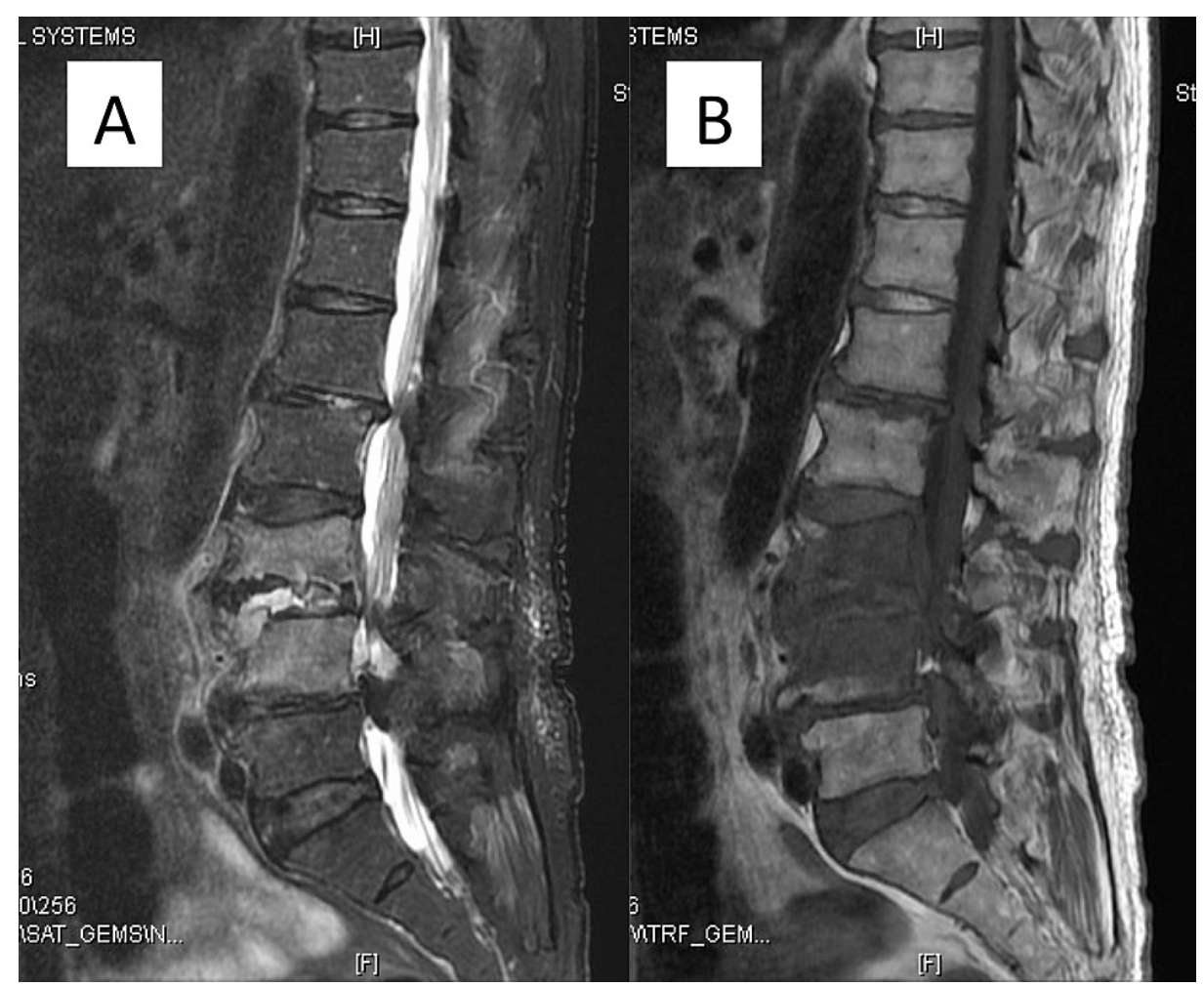

FIGURE 5: Secondary images obtained with magnetic resonance imaging.

(A) Sagittal T2-weighted fat-suppressed image. (B) Sagittal T1-weighted image. Fluid collection in the intervertebral disc space and diffuse signal changes in the L3 and L4 vertebral bodies with destructive changes were observed.

We simultaneously performed PPS fixation from T10 to the pelvis and PEDD to L3/4 at 34 days after admission. Bilateral PPS for T10 to L2 and L5, and S1 and bilateral S2-alar-iliac screws were inserted and percutaneously connected to the rod. Two other rods were fixed between the L2 to L5 level with lateral connectors to achieve a four-rod construct (Figure 6). The rod was bent in situ using an intraoperative spinal rod bending system (Bendini; NuVasive Inc., San Diego, CA). PEDD was performed via another incision. In the prone position, percutaneous endoscopic observation, debridement with forceps and a bipolar coagulator, and irrigation were performed for the L3/4 intervertebral disc. Instead of the normal nucleus pulposus, infectious muddy fluid and some floating tissue were observed and collected. A Blake silicon drainage tube (10-Fr, Blake drains and J-VAC Suction Reservoirs; Ethicon Inc., NJ) was placed in the disc space and fixed with a suture. 


\section{Cureus}

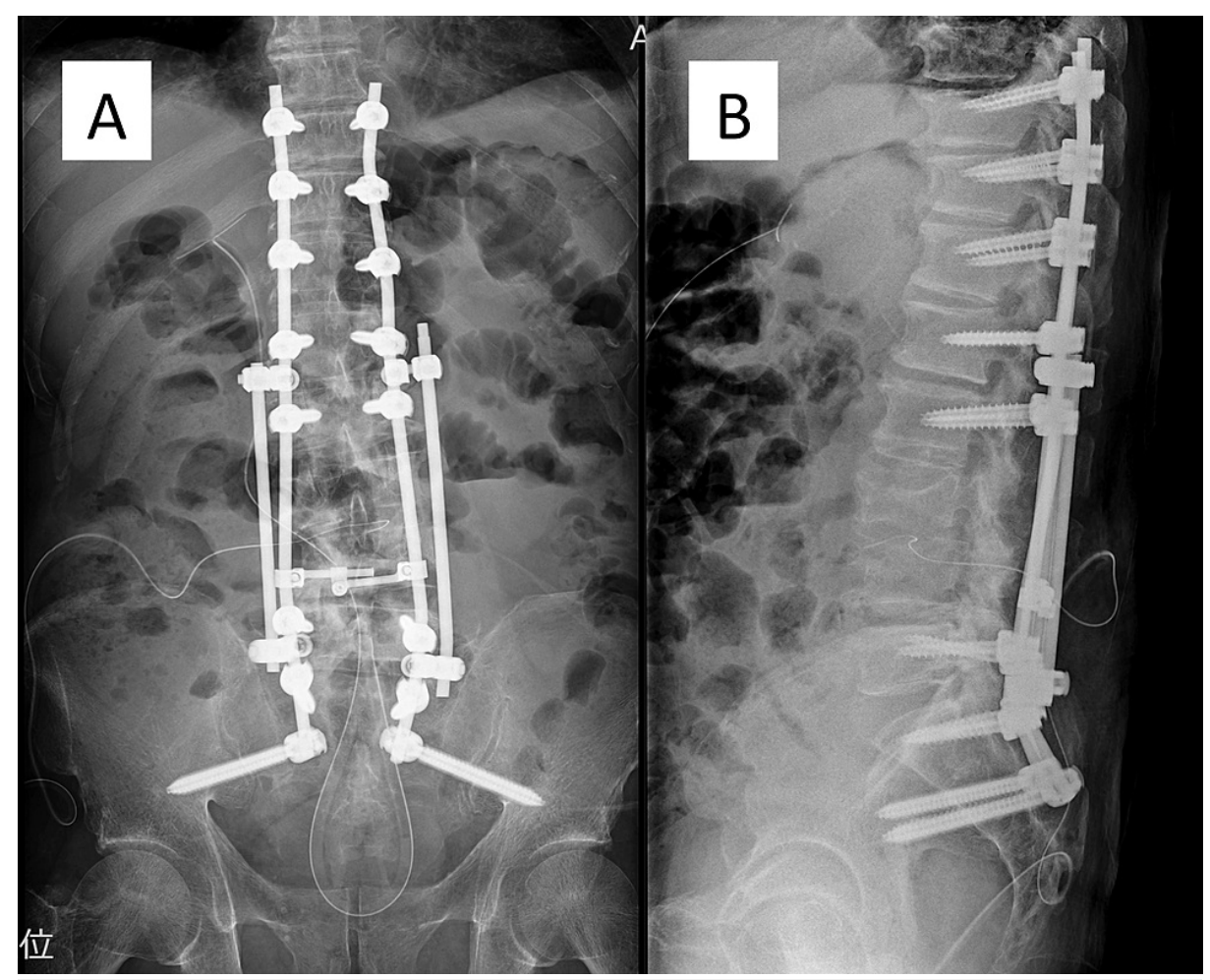

FIGURE 6: Postoperative X-ray images of posterior percutaneous screw fixation from T10 to the pelvis and percutaneous endoscopic discectomy/debridement to L3/4.

(A) Anteroposterior X-ray image. (B) Lateral X-ray image.

E. coli was detected again during a culture analysis of the intraoperatively obtained samples. The antibiotic regimen was changed to cefazolin (CEZ) 40 days after admission (Figure 1).

His back pain improved immediately and the drainage tube was removed 47 days after admission; however, oozing from the drainage tube wound continued. The fluid was initially serous and gradually changed to purulent fluid. Local debridement with local anesthesia was performed 55 days after admission; Corynebacterium amycolatum and Corynebacterium striatum were detected during a pus culture analysis. Secondary infection with multidrug-resistant bacteria was diagnosed. Teicoplanin (TEIC) administration was started immediately and CEZ administration was continued (Figure 1). We performed anterior debridement and autogenous bone grafting to L3/4 at 64 days after admission using the retroperitoneal approach because the focus of the secondary infection was considered to be located in the L3/4 disc space and the vertebral body of L3 and L4 (Figures 7, 8). At that point, PEDD from right side had already been performed and failed, and the posterior fusion was already performed, and we could not detect the undoubted reason for the secondary infection or microbial substitution. Therefore, we considered that another surgical option of re-PEDD from the other side might fail to control the infection. An autogenous bone graft was harvested from his right iliac crest. Debridement and suturing of the fistula were also performed. TEIC and CEZ were continued for six weeks after anterior debridement surgery. The CRP level turned negative at 14 weeks after admission. At the follow-up at 12 months after final surgery, the infection did not recur, CRP level stays negative, no spinal instrument failure/loosening and bone union around the autogenous bone graft were observed by CT scans, and the patient does not complain of any symptoms (Figures 1,9). 


\section{Cureus}

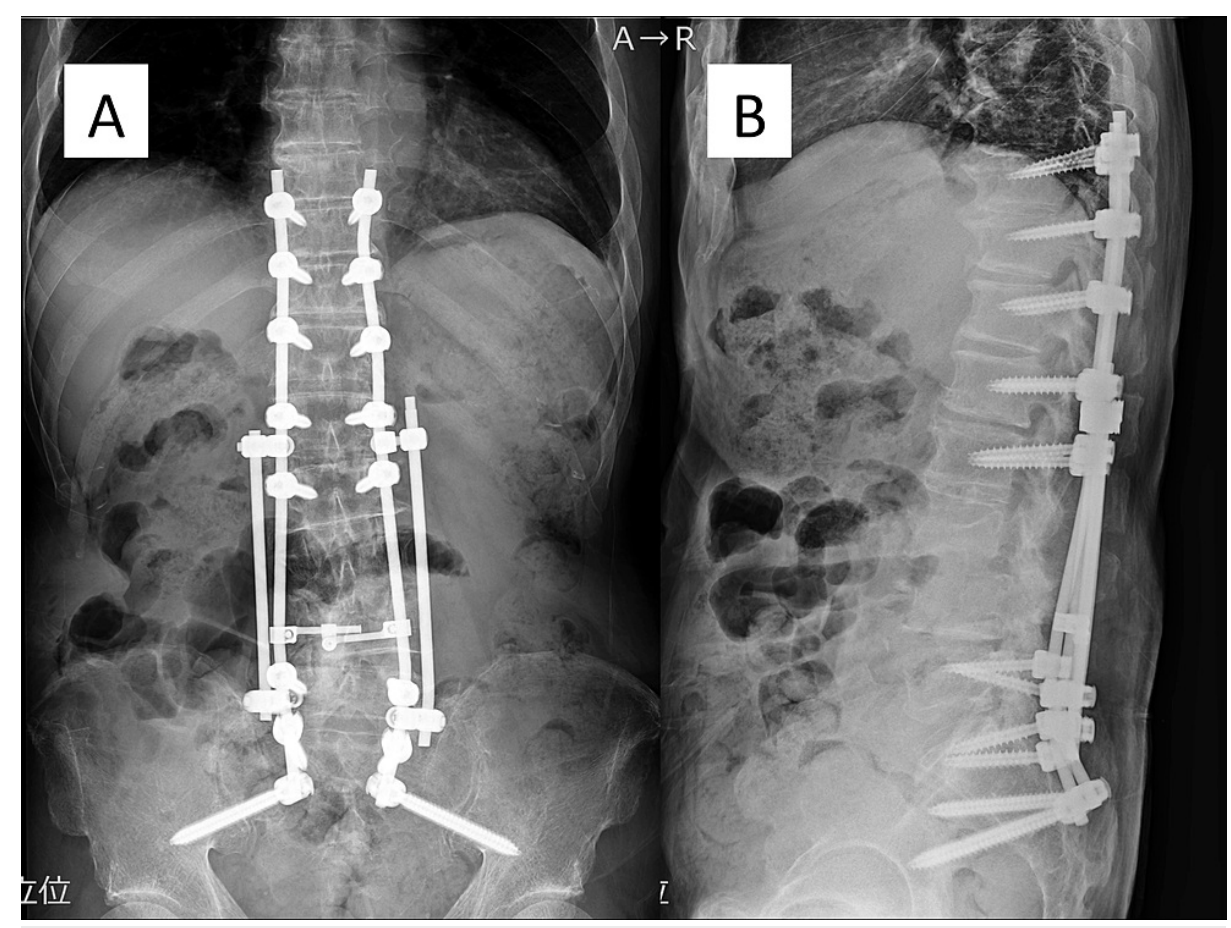

FIGURE 7: Postoperative X-ray of anterior debridement and autogenous bone grafting to $L 3 / 4$.

(A) Anteroposterior X-ray image. (B) Lateral X-ray image. 


\section{Cureus}

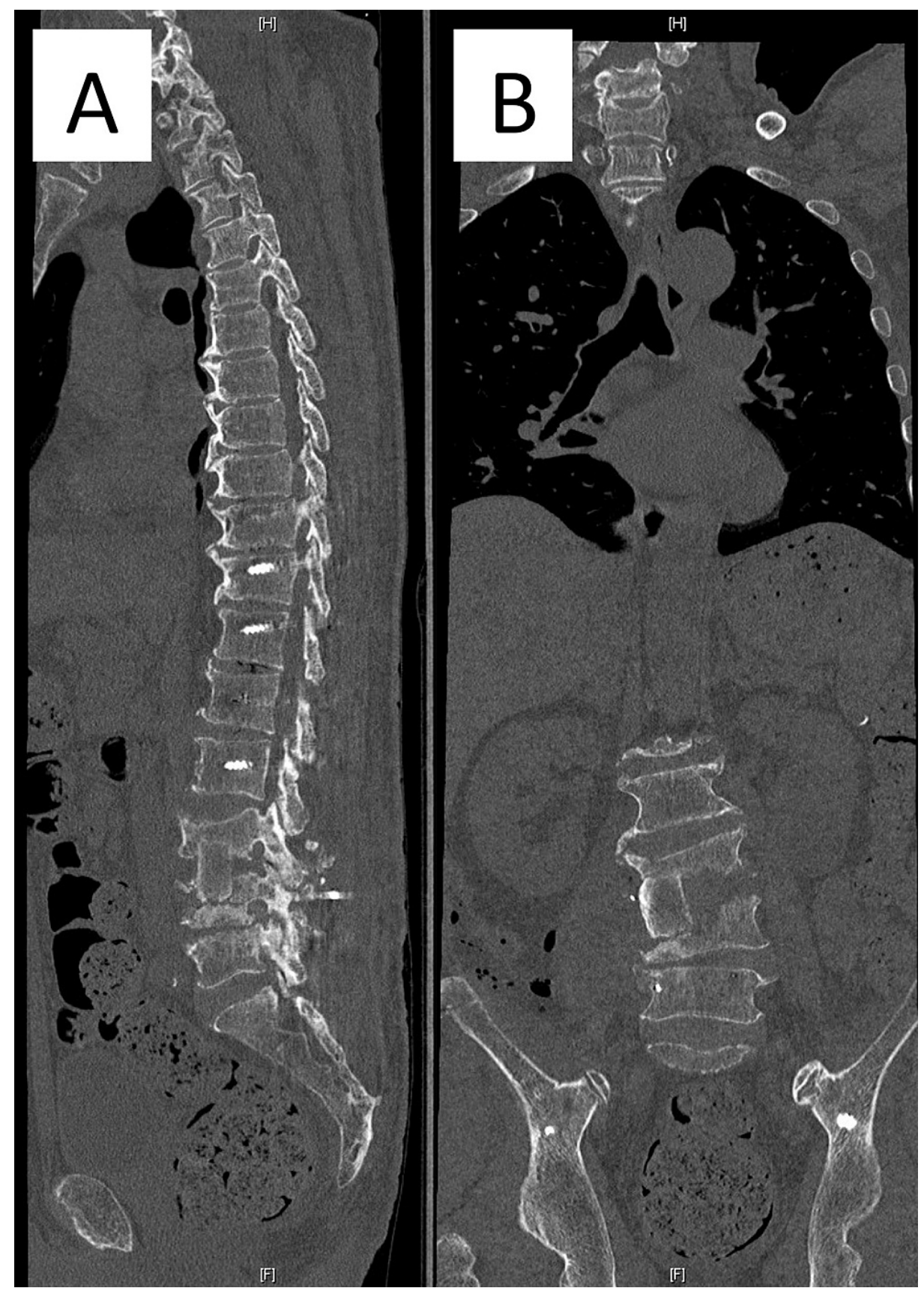

FIGURE 8: Postoperative computed tomography (CT) images of anterior debridement and autogenous bone grafting to $L 3 / 4$.

(A) Sagittal CT image. (B) Coronal CT image. 


\section{Cureus}

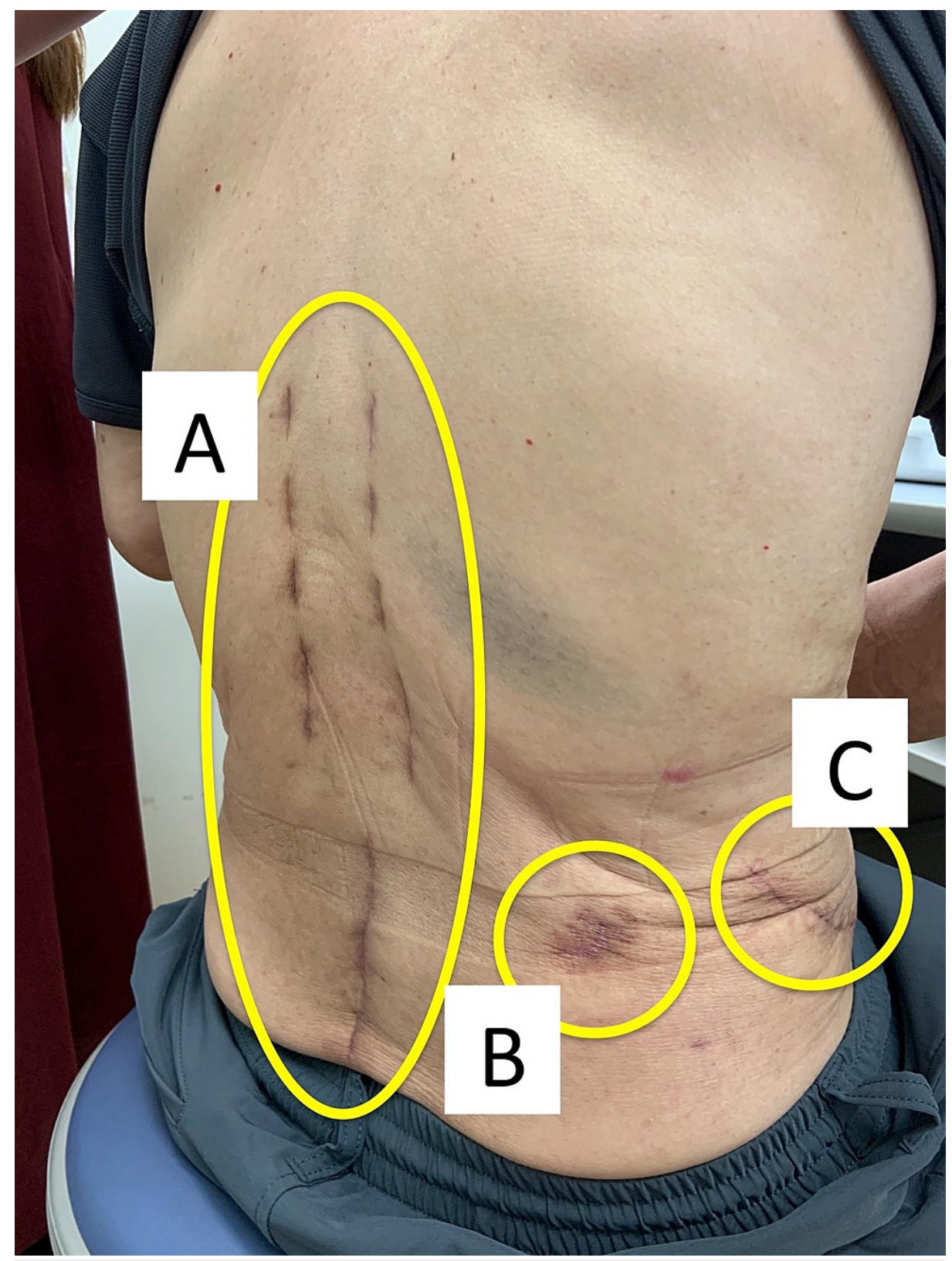

\section{FIGURE 9: Postoperative scars}

(A) Scars after posterior percutaneous pedicle screw fixation. (B) Scar after percutaneous endoscopic discectomy/debridement. (C) Scar after anterior debridement and autogenous bone grafting.

Written informed consent was obtained from the patient for publication of this case report. The study was performed in accordance with the Declaration of Helsinki and within the appropriate ethical framework.

\section{Discussion}

Our patient had a PS treated with PEDD, PPS fusion, anterior debridement, and autogenous bone grafting with characteristic oozing of pus. Multiple surgical procedures, rest, and antibiotics were required. The treatment strategy for PS comprises: identifying the pathogen [2,16]; appropriate antibiotic administration; posterior fusion to achieve local stability; and debridement, including needle puncture, percutaneous endoscopic lumbar discectomy/debridement (PEDD), and anterior debridement fusion.

To identify pathogens, which is the first important step, a blood culture analysis and biopsy of a sample obtained through PED or CT-guided biopsy before starting antibiotic administration are mandatory $[2,16]$. In this case, E. coli was detected during the blood culture analysis. Therefore, it was considered reasonable that PS was hematogenously induced/transferred by choledocholithiasis cholangitis. Intravenous antibiotic administration for six weeks is recommended $[2,17]$. However, surgical indications and standards for PS are 
still unclear. Options include posterior fusion, PPS fusion, anterior debridement/fusion, and PEDD.

When conservative treatment is ineffective and surgical procedures are inevitable, we first consider PEDD and/or PPS fusion; we secondarily consider anterior debridement and autogenous bone grafting. First of all, there is still no consensus on the optimal surgical strategy for PS [2]. Recently, the effectiveness of PPS fusion without anterior debridement is reported $[11,12,18,19]$; the effectiveness of intradiscal drainage with PEDD has been previously reported $[2,4,5,7-10,14,15,20]$. There is a recent article reported successful case series treated by one-stage posterior fixation with anterior debridement from posterior approach [21]. When infection focus is limited in anterior and middle column, we usually avoid the anterior interbody/intradiscal procedure via posterior approach because we worry about the iatrogenic infection progress to the posterior area. As a result, we had to perform every aforementioned surgical option because of the poor clinical course.

E. coli was the initial cause of PS in this case. A literature review indicated that PS caused by Gram-negative rod bacteria has good sensitivity to antibiotics and responds well to conservative treatment [22-26]. In this case, secondary infection with C. amycolatum and C. striatum caused an active infectious response in the L3/4 disc space and positive pressure from the focus of infection resulted in continuous oozing of pus through a tunnel formed in muscle and subcutaneous tissue in the portal route used for PEDD. In this case, retrograde infection via drain tube could be a potential cause of the secondary infection. Secondary infection/microbial substitution in PS should be considered when the clinical course worsens, although this condition is considered uncommon, and the cause and incidence are unknown.

\section{Conclusions}

We reported a case of lumbar pyogenic spondylitis treated with anterior debridement fusion after percutaneous endoscopic discectomy drainage with percutaneous pedicle screw fixation because of the continuous oozing of pus from the postoperative PEDD incision caused by secondary infection/microbial substitution. The possibility of secondary infection/microbial substitution in pyogenic spondylitis should be considered when the clinical course worsens.

\section{Additional Information}

\section{Disclosures}

Human subjects: Consent was obtained or waived by all participants in this study. Conflicts of interest: In compliance with the ICMJE uniform disclosure form, all authors declare the following: Payment/services info: All authors have declared that no financial support was received from any organization for the submitted work. Financial relationships: All authors have declared that they have no financial relationships at present or within the previous three years with any organizations that might have an interest in the submitted work. Other relationships: All authors have declared that there are no other relationships or activities that could appear to have influenced the submitted work.

\section{References}

1. Park KH, Kim DY, Lee YM, et al.: Selection of an appropriate empiric antibiotic regimen in hematogenous vertebral osteomyelitis. PLoS One. 2019, 14:e0211888. 10.1371/journal.pone.0211888

2. Berbari EF, Kanj SS, Kowalski TJ, et al.: Executive summary: 2015 Infectious Diseases Society of America (IDSA) clinical practice guidelines for the diagnosis and treatment of native vertebral osteomyelitis in adults. Clin Infect Dis. 2015, 61:859-63. 10.1093/cid/civ633

3. Hanaoka N, Kawasaki Y, Sakai T, et al.: Percutaneous drainage and continuous irrigation in patients with severe pyogenic spondylitis, abscess formation, and marked bone destruction. J Neurosurg Spine. 2006, 4:374-9. 10.3171/spi.2006.4.5.374

4. Kono M, Koda M, Abe T, et al.: Percutaneous endoscopic discectomy might be effective in selected cases of pyogenic spondylitis. J Orthop Surg (Hong Kong). 2019, 27: 10.1177/2309499019885446

5. Ito M, Abumi K, Kotani Y, Kadoya K, Minami A: Clinical outcome of posterolateral endoscopic surgery for pyogenic spondylodiscitis: results of 15 patients with serious comorbid conditions. Spine (Phila Pa 1976). 2007, 32:200-6. 10.1097/01.brs.0000251645.58076.96

6. Yang SC, Fu TS, Chen LH, Niu CC, Lai PL, Chen WJ: Percutaneous endoscopic discectomy and drainage for infectious spondylitis. Int Orthop. 2007, 31:367-73. 10.1007/s00264-006-0188-6

7. Fu TS, Chen LH, Chen WJ: Minimally invasive percutaneous endoscopic discectomy and drainage for infectious spondylodiscitis. Biomed J. 2013, 36:168-74. 10.4103/2319-4170.112742

8. Lin GX, Kim JS, Sharma S, et al.: Full endoscopic discectomy, debridement, and drainage for high-risk patients with spondylodiscitis. World Neurosurg. 2019, 127:202-11. 10.1016/j.wneu.2019.02.206

9. Fu TS, Yang SC, Tsai TT, Chen LH, Lai PL, Niu CC, Chen WJ: Percutaneous endoscopic debridement and drainage in immunocompromised patients with complicated infectious spondylitis. Minim Invasive Ther Allied Technol. 2010, 19:42-7. 10.3109/13645700903384450

10. Yang SC, Chen WJ, Chen HS, Kao YH, Yu SW, Tu YK: Extended indications of percutaneous endoscopic lavage and drainage for the treatment of lumbar infectious spondylitis. Eur Spine J. 2014, 23:846-53. 10.1007/s00586-013-3157-y

11. Lin TY, Tsai TT, Lu ML, et al.: Comparison of two-stage open versus percutaneous pedicle screw fixation in treating pyogenic spondylodiscitis. BMC Musculoskelet Disord. 2014, 15:443. 10.1186/1471-2474-15-443

12. Nasto LA, Colangelo D, Mazzotta V, et al.: Is posterior percutaneous screw-rod instrumentation a safe and 
effective alternative approach to TLSO rigid bracing for single-level pyogenic spondylodiscitis? Results of a retrospective cohort analysis. Spine J. 2014, 14:1139-46. 10.1016/j.spinee.2013.07.479

13. Deininger MH, Unfried MI, Vougioukas VI, Hubbe U: Minimally invasive dorsal percutaneous spondylodesis for the treatment of adult pyogenic spondylodiscitis. Acta Neurochir (Wien). 2009, 151:1451-7.

10.1007/s00701-009-0377-3

14. Sundararaj GD, Babu N, Amritanand R, Venkatesh K, Nithyananth M, Cherian VM, Lee VN: Treatment of haematogenous pyogenic vertebral osteomyelitis by single-stage anterior debridement, grafting of the defect and posterior instrumentation. J Bone Joint Surg Br. 2007, 89:1201-5. 10.1302/0301-620X.89B9.18776

15. Faciszewski T, Winter RB, Lonstein JE, Denis F, Johnson L: The surgical and medical perioperative complications of anterior spinal fusion surgery in the thoracic and lumbar spine in adults. A review of 1223 procedures. Spine (Phila Pa 1976). 1995, 20:1592-9. 10.1097/00007632-199507150-00007

16. Gouliouris T, Aliyu SH, Brown NM: Spondylodiscitis: update on diagnosis and management . J Antimicrob Chemother. 2010, 65:11-24. 10.1093/jac/dkq303

17. Rutges JP, Kempen DH, van Dijk M, Oner FC: Outcome of conservative and surgical treatment of pyogenic spondylodiscitis: a systematic literature review. Eur Spine J. 2016, 25:983-99. 10.1007/s00586-015-4318-y

18. Chen MJ, Niu CC, Hsieh MK, Luo AJ, Fu TS, Lai PL, Tsai TT: Minimally invasive transforaminal lumbar interbody debridement and fusion with percutaneous pedicle screw instrumentation for spondylodiscitis. World Neurosurg. 2019, 128:744-51. 10.1016/j.wneu.2019.04.249

19. Tschugg A, Hartmann S, Lener S, Rietzler A, Sabrina N, Thomé C: Minimally invasive spine surgery in lumbar spondylodiscitis: a retrospective single-center analysis of 67 cases. Eur Spine J. 2017, 26:3141-6. 10.1007/s00586-017-5180-x

20. Funayama T, Sakane M, Fujii K, Tsukanishi T, Noguchi H, Yamazaki M: Outcomes of percutaneous endoscopic debridement and drainage for septic spondylodiscitis with marked bone destruction. Internet J Spine Surg. 2014, 9:1-8.

21. Su B, Tang K, Liu W, Luo X, Quan Z, Jiang D, Peng X: One-stage posterior debridement, autogenous spinous process bone graft and instrumentation for single segment lumbar pyogenic spondylitis. Sci Rep. 2021, 11:3065. 10.1038/s41598-021-82695-2

22. Kang SJ, Jang HC, Jung SI, et al.: Clinical characteristics and risk factors of pyogenic spondylitis caused by Gram-negative bacteria. PLoS One. 2015, 10:e0127126. 10.1371/journal.pone.0127126

23. Park KH, Cho OH, Jung M, et al.: Clinical characteristics and outcomes of hematogenous vertebral osteomyelitis caused by Gram-negative bacteria. J Infect. 2014, 69:42-50. 10.1016/j.jinf.2014.02.009

24. Lee CY, Wu MH, Cheng CC, et al.: Comparison of Gram-negative and gram-positive hematogenous pyogenic spondylodiscitis: clinical characteristics and outcomes of treatment. BMC Infect Dis. 2016, 16:735. 10.1186/s12879-016-2071-4

25. Graham SM, Fishlock A, Millner P, Sandoe J: The management gram-negative bacterial haematogenous vertebral osteomyelitis: a case series of diagnosis, treatment and therapeutic outcomes. Eur Spine J. 2013, 22:1845-53. 10.1007/s00586-013-2750-4

26. Del Pozo FJ, Alonso JV, Ruiz MÁ, Vythilingam S, Ruiz DL: Community acquired spondylodiscitis caused by Escherichia coli; case report and literature review. Bull Emerg Trauma. 2016, 4:174-9. 